\title{
Lateral contact of slender prismatic bodies
}

\author{
By José Castillo and J. R. Barber \\ Department of Mechanical Engineering and Applied Mechanics, \\ University of Michigan, Ann Arbor, MI 48109-2125, USA
}

Many nominally two-dimensional contact problems, such as the contact of parallel rollers or spur gears, exhibit slow axial variation in contact conditions due to manufacturing errors, misalignment or generalized beam deflections of the contacting bodies. In this paper, an asymptotic method is used to develop a solution to such problems, the inner problem being the two-dimensional Hertzian contact theory and the outer problem the bending of the contacting bodies as beams separated by a nonlinear elastic foundation.

The method is illustrated in the case of two cylindrical rollers that are slightly misaligned and pressed together by forces applied at their ends. The classical Hertzian elliptical contact area is obtained at low loads, but with increasing load the contact area evolves first into a dog-bone shape and then into two separated contact areas surrounding the point forces that would be predicted by an elementary beam theory solution of the problem.

\section{Introduction}

The classical two-dimensional Hertzian contact theory forms the basis of the analysis of a wide range of important engineering components, including parallel roller bearings and spur gears. However, there are many cases in which the contact conditions and the associated stress fields vary, albeit slowly, along the contact axis. For example, spalling failures of spur gears hardly ever occur uniformly along the nominal contact length, but are concentrated in a region near one end - a sure sign of nonuniform contact (Juvinall 1983). This could be due to a slight bearing misalignment or machining errors, though a more likely source of non-uniformity is the bending of the shaft on which the gears are mounted due to contact loads. Other applications leading to slow axial variation of nominally two-dimensional contact stress fields include parallel roller bearings in which one roller becomes slightly misaligned (Barber 1980) or in which the roller diameter varies slightly along the axis.

Even if the contacting bodies are uniform and perfectly aligned, bending deflections can lead to non-uniformity along the axis. For example, if the two contacting rolls of a rolling mill are of uniform diameter and are pressed together by bearing forces at the ends, uniform contact pressure along the axis can only be ensured if the rolls are slightly crowned - i.e. of larger diameter near the centre than at the ends - in order to compensate for bending deflections under the influence of the uniform load. The required crown is typically calculated using bending theory and will vary depending on the particular strip rolling process. This variability can be accommodated by using a different set of rolls for each process, but a more efficient procedure is to

Proc. R. Soc. Lond. A (1997) 453, 2397-2412

Printed in Great Britain

(C) 1997 The Royal Society $\mathrm{T}_{\mathrm{E} X}$ Paper 
use continuously variable crown (CVC) rolls, in which the effective crown can be changed by making a small axial relative motion of the mating rolls (McDonald \& Spooner 1989). The problem is complicated by the fact that the rolls will expand due to frictional and/or workpiece heating, giving a superposed 'thermal crown' (Bennon 1985).

Other applications exhibiting similar behaviour include cases where a long cylindrical bar is embedded in, but not bonded to, an extended medium and is then subjected to lateral loading - for example, a vertical embedded pile subjected to horizontal loading at its free end (Liu 1990), or a steel pin embedded in bone in orthopedic applications. In these cases, a contact problem arises when the lateral loading is sufficient to cause the embedded bar to separate from the medium in some regions.

Kalker $(1972,1977)$ developed an asymptotic method of approximating the solution of three-dimensional problems for the half-space when the contact area has a large aspect ratio and conditions vary relatively slowly along the longer dimension. Two length scales are defined associated with typical width and length dimensions of the contact area, respectively. On the scale of the (small) contact width, the variation in conditions along the axis is so slow that the fields are locally almost two dimensional. By contrast, on the scale of the length of the contact area, the contact width is very small and the fields approximate those associated with a varying line load.

Kalker's method applies to contact problems for the half-space; i.e. the semiinfinite body bounded by a plane. Of course, real bodies have finite dimensions, but a half-space model is appropriate if these are large in comparison with the dimensions of the contact area-including the relatively long axial dimension. However, most of the applications discussed above fail this test. For example, the width of the contact area between two misaligned spur gears will be small compared with the tooth thickness, but the longer axial dimension of the contact area will not. This has two important consequences for the analysis: (i) the local two-dimensional field will now have to be determined for a finite body consisting of a 'slice' of the original threedimensional prismatic body; and (ii) bending of the prismatic body as a beam will affect the relative kinematics of different slices and hence couple the axial variation of the contact conditions and the beam-bending problem.

In this paper, we shall give a theoretical derivation appropriate to problems of this class and illustrate its use by examples in which the contacting bar has a circular cross-section. In essence, the method reduces to the solution of a problem for the bending of a beam on a nonlinear elastic foundation, the characteristics of the foundation being determined by the solution of the two-dimensional 'slice' theory for the local contact conditions.

In particular, we shall show that the half-space assumption embedded in the classical Hertz theory leads to significant errors when the axial semi-axis of the predicted contact area is larger than the radius of the bar and that, at larger loads, the Hertzian theory fails to describe even the qualitative nature of the evolution of the contact area.

\section{Features of the three-dimensional stress field}

Consider the problem illustrated in figure 1, in which a circular cylindrical bar of radius $R$ is pressed against a rigid body, establishing a contact area whose half-width,

Proc. R. Soc. Lond. A (1997) 


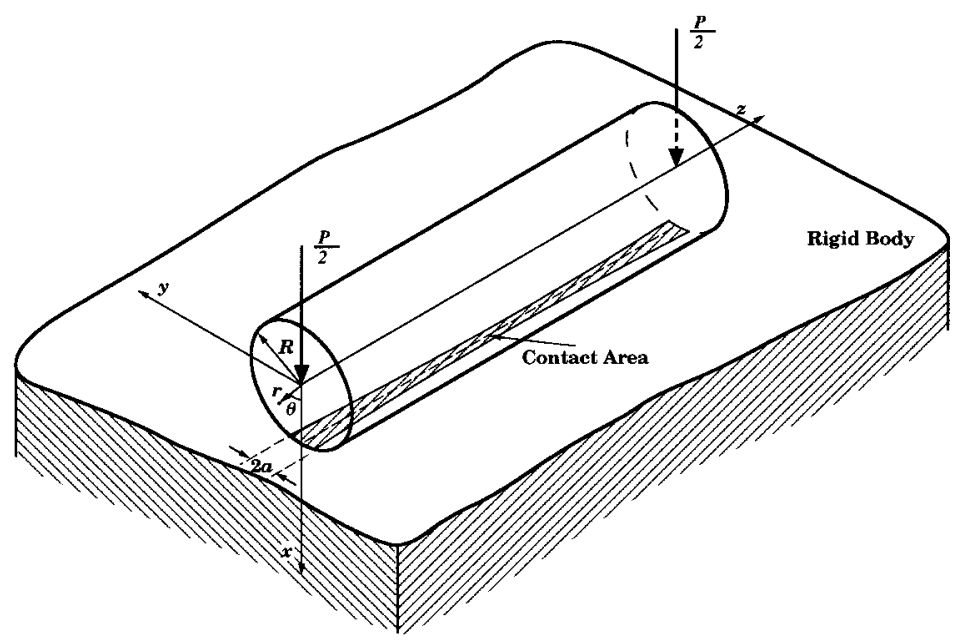

Figure 1. Contact between an elastic cylinder and a rigid body.

$a$, is a function of axial position $z$. The contact will result in tractions applied to the bar whose force resultant $F$ per unit length is also a function of $z$. We define a cylindrical polar coordinate system $r, \theta$, such that the centre of the contact region is identified with the point $(R, 0)$.

The contact load will cause a shear force $V$ and a bending moment $M$ in the bar and corresponding bending and shear deflections. If the variation in $a$ is fairly slowmore precisely, if $\mathrm{d} a / \mathrm{d} z \ll a / R$-we anticipate that the in-plane stresses $\sigma_{r r}, \sigma_{r \theta}, \sigma_{\theta \theta}$ will approximate the solution of an appropriate plane strain two-dimensional problem, whilst the out-of-plane stresses $\sigma_{z r}, \sigma_{z \theta}, \sigma_{z z}$ will depend upon $V$ and $M$.

In general, this will be only an approximate statement, but there are cases in which an exact solution can be written down in the context of three-dimensional elasticity. For example, consider the case in which $F$ and $a$ are independent of $z$; i.e. the beam is subjected to a uniformly distributed load, so that $V$ is a linear function and $M$ a quadratic function of $z$. We can construct the solution to this problem by superposing: (i) the three-dimensional solution for a circular cylinder loaded by a uniform body force and supported at its ends (see Love 1927, §244); and (ii) the plane strain problem of the same bar loaded by an equal and opposite uniform body force and supported by Hertzian contact stresses along a strip of uniform width $2 a$. This solution is not given here in the interests of brevity, but it is important to note that the only contribution to the out-of-plane shear stresses $\sigma_{z r}, \sigma_{z \theta}$ comes from solution (i) and these have the same distribution as is obtained in the classical bending problem of a cantilever beam with an end load. In Cartesian coordinates $x=r \cos \theta, y=r \sin \theta$, we have

$$
\sigma_{z x}=V f_{x}(x, y), \quad \sigma_{z y}=V f_{y}(x, y)
$$

where

$$
\begin{aligned}
& f_{x}(x, y)=\frac{1}{2 \pi(1+\nu) R^{4}}\left[(3+2 \nu)\left(R^{2}-x^{2}\right)-(1-2 \nu) y^{2}\right] \\
& f_{y}(x, y)=-\frac{(1+2 \nu) x y}{\pi(1+\nu) R^{4}}
\end{aligned}
$$

and $\nu$ is Poisson's ratio (see Timoshenko \& Goodier 1970, $\S 122$ ). We consider $f_{x}, f_{y}$ 
as the components of a two-dimensional vector field $\boldsymbol{f}$. The reason for the similarity in form between these solutions is that the solution for the end-loaded cantilever can itself be constructed by superposing two equal and opposite uniform load solutions with a small axial displacement; i.e. by differentiating the stress and displacement fields for the uniformly loaded case with respect to $z$ (differentiation is of course a form of superposition here). It follows that the out-of-plane shear stress distribution for a beam with arbitrary cross-section and general, but axially uniform lateral loading can be determined by solving an equivalent end loading problem for the same cross-section, using the methods of ch. 12 of Timoshenko \& Goodier (1970).

\section{Relation to the beam theory}

In most applications, the contact conditions will vary along the axis and the above solution is strictly inapplicable. However, in the spirit of the theory of beams, it is reasonable to anticipate that the distribution of out-of-plane shear stress will be approximated by equations (2.1)-(2.3) as long as the load, $F$, varies only relatively slowly along the axis.

The beam theory demands that we give a unique meaning to the deflection at any cross-section, whereas the two-dimensional analysis describing the local contact conditions involves displacements that vary continuously through the cross-section. This problem is also encountered when imposing displacement boundary conditions in elasticity solutions. Renton $(1991,1996)$ argues that the only self-consistent definition of beam deflection is that which ensures that reactions imposed in the form of tractions distributed according to the appropriate St Venant theory do no work.

For a circular beam deflecting in the $x z$-plane, the appropriate St Venant traction distribution is that given by equations (2.1)-(2.3) and the resulting definition of beam deflection $U$ in the $x$-direction is therefore

$$
U=\iint_{A} \boldsymbol{f} \cdot \boldsymbol{u} \mathrm{d} A,
$$

where the integral is taken over the area $A=\pi R^{2}$ of the cross-section and $\boldsymbol{u}$ is the displacement vector in the two-dimensional solution.

\section{The two-dimensional problem}

We now turn our attention to the two-dimensional plane strain problem describing the in-plane stresses and displacements of a typical thin slice cut transversely from the beam. The frictionless Hertzian contact tractions (Johnson 1985) can be defined through the boundary conditions

$$
\begin{aligned}
\sigma_{r r} & =-\frac{2 F}{\pi a^{2}} \sqrt{a^{2}-R^{2} \sin ^{2} \theta}, \quad r=R, \quad-\psi<\theta<\psi, \\
\sigma_{r r} & =0, \quad r=R, \quad|\theta|>\psi, \\
\sigma_{r \theta} & =0, \quad r=R, \quad \text { all } \theta,
\end{aligned}
$$

where the contact semi-arc $\psi$ is defined by

$$
R \sin \psi=a=\sqrt{\frac{2 F R(1-\nu)}{\pi \mu}}
$$

and $\mu$ is the shear modulus for the material.

Proc. R. Soc. Lond. A (1997) 
The resultant force, $F$, will be balanced by differences between the values of $\sigma_{x z}, \sigma_{y z}$ on the two plane faces. Since the slice is taken to be thin, these balancing tractions can be replaced by body forces and they will be distributed according to the expressions in equations (2.1)-(2.3); i.e.

$$
\boldsymbol{p}=F \boldsymbol{f} .
$$

The two-dimensional problem is completely defined by equations (4.1)-(4.5). Notice that we do not require a full solution of this two-dimensional problem. The only quantity of interest is the difference

$$
U-\boldsymbol{i} \cdot \boldsymbol{u}(A),
$$

between the $x$-component of the displacement of the point $A(R, 0)$ ( $\boldsymbol{i}$ is defined as the unit vector in the $x$-direction) and the weighted average displacement $U$, which is the corresponding displacement of the cylinder considered as a beam defined in equation (3.1). The expression (4.6) will depend in a nonlinear way on $F$ (because the contact semi-arc $\psi$ depends on $F$ ) and it defines a compliance interposed between the rigid plane and the beam.

The more general case where the contact is conforming-i.e. where the cylinder makes contact with a groove or hole whose radius is not too different from $R$-can also be defined in this way, except that the Hertzian contact tractions must then be replaced by the expressions defined by Persson (1964) for a disk contacting a nearly conforming hole (see also Liu 1990 and Johnson 1985).

\section{(a) Solution}

The solution is conveniently constructed by superposition. We start with the classical plane elasticity problem of a uniform heavy disk supported by a concentrated force (Love 1927, $\S 155$ (iii)), as shown in figure $2 a$. Convolution of this solution on the pressure distribution of equations (4.1), (4.2) will then yield the solution for the two-dimensional problem, except that the desired body force distribution (4.5) will be replaced by a uniform distribution $p_{x}=F / \pi R^{2}, p_{y}=0$ with the same resultant force. Finally, we superpose a corrective solution, in which the disk is loaded by body forces equal to the difference between (4.5) and the uniform distribution and no boundary tractions.

(i) The heavy disk

The solution of the problem of figure $2 a$ is well known. It can be obtained by superposition of the Flamant solution for the concentrated force $F$ applied to a half plane (Barber 1992, § 12.3), a balancing uniform body force (Barber 1992, § 7.2.1) and some corrective terms taken from table 8.1 of Barber (1992) to satisfy the condition that the disk surface be traction free except at the point of application of the force. The appropriate Airy stress function is

$$
\phi=-\frac{F s \beta \sin (\beta)}{\pi}+\frac{F r^{2}}{4 \pi R}+\frac{F r^{3} \cos (\theta-\alpha)}{4 \pi R^{2}},
$$

with the body force potential

$$
V=-\frac{F r \cos (\theta-\alpha)}{\pi R^{2}} .
$$

Notice that the Flamant solution term in $\phi$ is conveniently expressed in terms of the polar coordinates $(s, \beta)$ centred on the point $B$.

Proc. R. Soc. Lond. A (1997) 
(a)
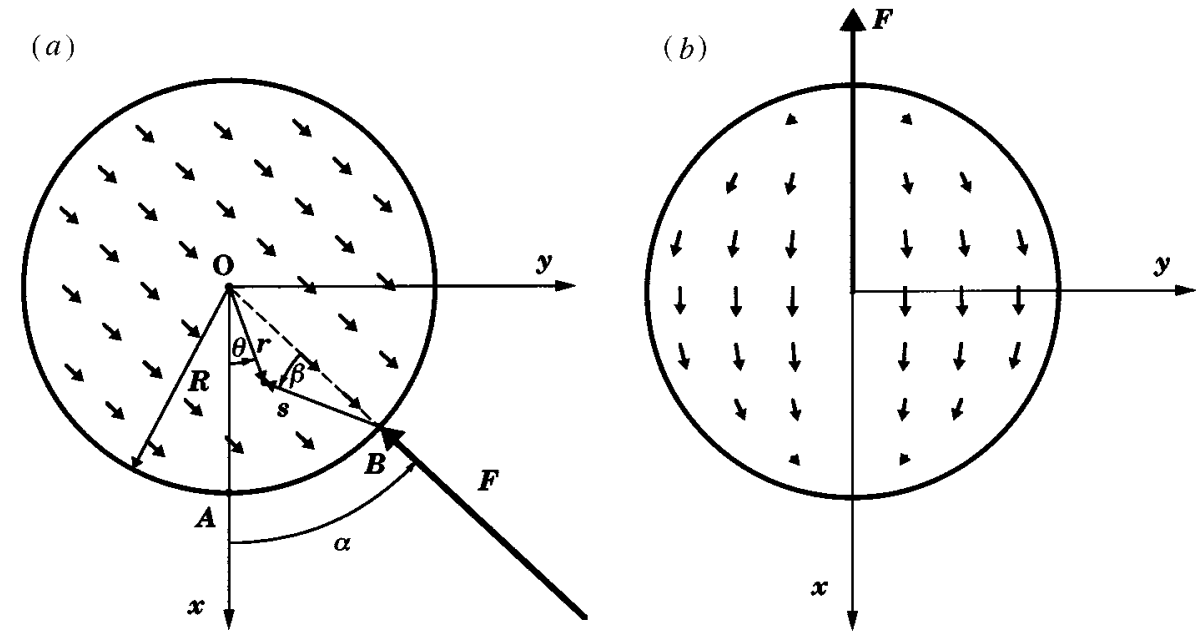

Figure 2. (a) A disk with uniform body force supported by a concentrated force at the edge; (b) auxiliary problem for use of Betti's reciprocal theorem.

The full displacement field for this problem can be found and substituted into equations (3.1), (4.6) to determine the difference between the weighted average 'beam' deflection $U$ and that at the point $A$ on the surface of the disk. However, the resulting integrals are very tedious because of the terms involving $(s, \beta)$ centred on the point $B$. A simpler approach is to apply Betti's reciprocal theorem to the problem, using as auxiliary solution the problem of figure $2 b$, in which a unit concentrated force at the origin is balanced by body forces of the form given in equation (4.5). The reciprocal theorem then states that

$$
\sum \boldsymbol{T}_{b} \cdot \boldsymbol{u}_{a}=\sum \boldsymbol{T}_{a} \cdot \boldsymbol{u}_{b}
$$

where $\boldsymbol{T}_{\gamma}, \boldsymbol{u}_{\gamma}$ are the tractions and displacements, respectively, in problem $\gamma$, $(\gamma=a, b)$.

In view of the definition of problem $(b)$, the left-hand side of equation (4.9) reduces to $U_{a}-\boldsymbol{i} . \boldsymbol{u}_{a}(O)$; i.e. the difference between the $x$-component of the central displacement and the beam displacement for problem $(a)$. Thus, the required displacement for problem (a) (expression (4.6) above) can be written

$$
\begin{aligned}
U_{a}-\boldsymbol{i} \cdot \boldsymbol{u}_{a}(A) & =\boldsymbol{i} \cdot\left(\boldsymbol{u}_{a}(O)-\boldsymbol{u}_{a}(A)\right)+\sum \boldsymbol{T}_{a} \cdot \boldsymbol{u}_{b} \\
& =\boldsymbol{i} \cdot\left(\boldsymbol{u}_{a}(O)-\boldsymbol{u}_{a}(A)\right)-\frac{1}{\pi R^{2}} \int_{0}^{2 \pi} \int_{0}^{R}\left(\boldsymbol{u}_{b} \cdot \boldsymbol{F}\right) r \mathrm{~d} r \mathrm{~d} \theta+\boldsymbol{F} \cdot \boldsymbol{u}_{b}(B) .
\end{aligned}
$$

Thus, the only displacement information required from problem $(a)$ is the difference between the $x$-components of displacement at $A$ and $O$, which can be obtained by routine methods (see, for example, Barber 1992, ch. 9) as

$$
\begin{aligned}
\boldsymbol{i} .\left(\boldsymbol{u}_{a}(O)-\boldsymbol{u}_{a}(A)\right)=\frac{F}{4 \pi \mu}\left[(1-2 \nu)(\pi-|\alpha|) \sin |\alpha|-\left\{4(1-\nu) \ln \left(2 \sin \left|\frac{1}{2} \alpha\right|\right)+\frac{1}{2}\right\}\right. \\
\times \cos (\alpha)-2(1-\nu)], \quad-\pi<\alpha<\pi .
\end{aligned}
$$

Proc. R. Soc. Lond. A (1997) 
The integral in equation (4.10) is considerably simpler than that in equation (3.1) because the displacement components in the auxiliary solution $(b)$ can be shown to have simple sinusoidal form, centred on $O$.

\section{(ii) The auxiliary solution}

The body force distribution (4.5) does not represent a conservative vector field and hence cannot be written as the gradient of a scalar body force potential. However, the solution to the problem of figure $2 b$ can be obtained by adding: (i) the Kelvin solution (Barber 1992, §13.1) describing the central force; and (ii) the general polynomial displacement field

$$
u_{r}=\left(C_{1} r^{4}+C_{2} r^{2}\right) \cos \theta, \quad u_{\theta}=\left(C_{3} r^{4}+C_{4} r^{2}\right) \sin \theta,
$$

where $C_{1}, C_{2}, C_{3}, C_{4}$ are arbitrary constants chosen so that the corresponding stresses satisfy the equilibrium equations and the traction-free boundary condition. After tedious but routine calculations, we obtain the displacement field

$$
\begin{aligned}
u_{r}=\frac{1}{96 \pi \mu\left(1-\nu^{2}\right)}[ & 12(1+\nu)(3-4 \nu) \ln (r)-6(1+\nu) \\
& \left.-\frac{4\left(7-9 \nu-14 \nu^{2}+4 \nu^{3}\right) r^{2}}{R^{2}}+\frac{\left(5-7 \nu-8 \nu^{2}\right) r^{4}}{R^{4}}\right] \cos \theta \\
u_{\theta}=-\frac{1}{96 \pi \mu\left(1-\nu^{2}\right)}[12(1+\nu)(3-4 \nu) \ln (r)+6(1+\nu) & \\
- & \left.\frac{4\left(1-7 \nu+6 \nu^{2}-4 \nu^{3}\right) r^{2}}{R^{2}}+\frac{\left(1-11 \nu+8 \nu^{2}\right) r^{4}}{R^{4}}\right] \sin \theta
\end{aligned}
$$

Substituting these expressions into (4.10), performing the integrations and using (4.11), we obtain

where

$$
U_{a}-\boldsymbol{i} \cdot \boldsymbol{u}_{a}(A)=\frac{F}{8 \pi \mu} g(\alpha)
$$

$$
\begin{aligned}
g(\alpha)= & 2(1-2 \nu)(\pi-|\alpha|) \sin |\alpha|-4(1-\nu) \\
& -\left\{8(1-\nu) \ln \left(2 \sin \left|\frac{1}{2} \alpha\right|\right)+\frac{2+3 \nu+4 \nu^{2}}{3(1+\nu)}\right\} \cos (\alpha), \quad-\pi<\alpha<\pi .
\end{aligned}
$$

(iii) The Hertzian solution

Equation (4.15) defines the required displacement difference for the case where the disk is subjected to a concentrated force at the point $(R, \alpha)$ on the boundary and a uniform equilibrating body force. The corresponding case where the force is distributed according to equations (4.1), (4.2), but the equilibrating body force is still uniform and can be written down by convolution as

$$
U_{a}-\boldsymbol{i} . \boldsymbol{u}_{a}(A)=\frac{F}{4 \pi^{2} \mu \sin ^{2} \psi} \int_{-\psi}^{\psi} g(\alpha) \sqrt{\sin ^{2} \psi-\sin ^{2} \alpha} \mathrm{d} \alpha,
$$

where we have used (4.4) to eliminate the contact semi-width $a$.

\section{(iv) Corrective solution}

Finally we need to superpose a corrective solution to adjust the body force from a uniform distribution to that defined by equation (4.5). This corresponds to the disk

Proc. R. Soc. Lond. A (1997) 
loaded by the difference between these loads, i.e.

$$
\boldsymbol{p}=F\left(\boldsymbol{f}-\frac{\boldsymbol{i}}{\pi R^{2}}\right),
$$

and with traction-free surfaces. The displacement field can be written in the form of equations (4.12), the four constants being again chosen so that the corresponding stress field satisfies: (i) the equilibrium condition with the required body force distribution; and (ii) the traction-free boundary condition. The calculations are routine and the correction to the relative displacement is found to be

$$
U_{\mathrm{c}}-\boldsymbol{i} \cdot \boldsymbol{u}_{\mathrm{c}}(A)=\frac{F(1-2 \nu)\left(107+211 \nu-84 \nu^{2}-224 \nu^{3}\right)}{2304 \pi \mu(1-\nu)(1+\nu)^{2}},
$$

where the suffix c denotes the corrective solution.

\section{(b) Results}

The complete solution of the two-dimensional problem is found by adding equations (4.17) and (4.19). As long as $\psi \ll 1$, the integral in (4.17) can be performed in closed form. We obtain

$$
U-U_{A}=U_{a}-\boldsymbol{i} \cdot \boldsymbol{u}_{a}(A)+U_{\mathrm{c}}-\boldsymbol{i} \cdot \boldsymbol{u}_{\mathrm{c}}(A) \equiv \frac{F h(\psi)}{\pi \mu},
$$

where

$$
h(\psi)=-(1-\nu) \ln \left(\frac{1}{2} \psi\right)-\frac{85+291 \nu+698 \nu^{2}-232 \nu^{3}-832 \nu^{4}}{2304(1-\nu)(1+\nu)^{2}}+O\left(\psi^{3}\right),
$$

$U$ is the beam deflection as defined in equation (3.1) and $U_{A} \equiv \boldsymbol{i} \cdot \boldsymbol{u}_{A}$ is the $x$ component of displacement of the point $A$ at the centre of the contact area.

For larger values of $\psi$, the contact pressure distribution will deviate from (4.1) (see, for example, Persson 1964) and the convolution integral would have to be performed numerically. This case is not pursued in the present paper.

\section{The beam problem}

The results of the previous section can now be incorporated into a solution for the beam deflection, $U$, yielding a problem for a beam on a nonlinear elastic foundation. The force $F$ now represents the load on the beam per unit length and hence the governing equation, including shear displacements, can be written

$$
\frac{\mathrm{d}^{4} U}{\mathrm{~d} z^{4}}+\frac{1}{K_{\mathrm{s}}} \frac{\mathrm{d}^{2} F}{\mathrm{~d} z^{2}}-\frac{F}{E I}=0,
$$

where $E I, K_{\mathrm{s}}$ are the flexural rigidity and the shear stiffness of the beam, respectively, (Pilkey 1994). We then substitute for $F$ from equation (4.20) and use $I=\frac{1}{4} \pi R^{4}$, and Renton's expression (Renton 1991) for $K_{\mathrm{s}}$ for the circular cross-section of radius $R$. After some simplifications, we obtain

$$
\frac{\mathrm{d}^{4} U}{\mathrm{~d} Z^{4}}+C_{1} \frac{\mathrm{d}^{2}}{\mathrm{~d} Z^{2}}\left(\frac{U}{h(\psi)}\right)-\frac{C_{2} U}{h(\psi)}=C_{1} \frac{\mathrm{d}^{2}}{\mathrm{~d} Z^{2}}\left(\frac{U_{A}}{h(\psi)}\right)-\frac{C_{2} U_{A}}{h(\psi)},
$$

where the dimensionless axial coordinate $Z=z / R$ and

$$
C_{1}=\frac{1}{6}\left(7+\left(\frac{\nu}{1+\nu}\right)^{2}\right), \quad C_{2}=\frac{2}{(1+\nu)} .
$$

Proc. R. Soc. Lond. A (1997) 
In equation (5.2), the displacement $U_{A}$ will be a known function of $z$ if the cylindrical beam is pressed against a rigid body of known shape. The nonlinearity of the equation is hidden in the dependence of $\psi$ and hence $h(\psi)$ on $F(Z)$. However, this dependence is relatively weak because of the logarithm in equation (4.21), suggesting that an iterative procedure would be convergent.

It is worth noting that there is one special case where the governing equation is strictly linear. If the beam is an exact fit inside a conforming hole, the problem is one of receding contact as defined by Dundurs (1975). In this case, the contact arc is unaffected by $F$. Even if $F$ becomes negative, an equal contact arc will occur on the other side of the support, so the problem becomes exactly that of a beam on a linear elastic foundation. If the cylinder is a force fit inside a circular hole, the problem will be linear to loads that are insufficient to cause separation from the support, but the foundation will then have a softening characteristic, approaching a limiting stiffness equal to that of the conforming hole at large loads. If the cylinder is a loose fit, the stiffness increases from zero at zero load to the same conforming cylinder limit at large loads.

\section{(a) Numerical solution}

To solve equation (5.2), we first integrate four times with respect to $Z$, obtaining

$$
\begin{aligned}
U+C_{1} & \int_{0}^{Z} \int_{0}^{Z} \frac{U}{h} \mathrm{~d} Z \mathrm{~d} Z-C_{2} \int_{0}^{Z} \int_{0}^{Z} \int_{0}^{Z} \int_{0}^{Z} \frac{U}{h} \mathrm{~d} Z \mathrm{~d} Z \mathrm{~d} Z \mathrm{~d} Z \\
= & U(0)+Z U^{\prime}(0)-\frac{1}{2}\left(C_{2} R Z^{2}\right) M^{*}(0)-\frac{1}{6}\left(C_{2} R Z^{3}\right) V^{*}(0) \\
& +C_{1} \int_{0}^{Z} \int_{0}^{Z} \frac{U_{A}}{h} \mathrm{~d} Z \mathrm{~d} Z-C_{2} \int_{0}^{Z} \int_{0}^{Z} \int_{0}^{Z} \int_{0}^{Z} \frac{U_{A}}{h} \mathrm{~d} Z \mathrm{~d} Z \mathrm{~d} Z \mathrm{~d} Z,
\end{aligned}
$$

where

$$
V^{*}=\frac{V}{\mu \pi R^{2}} \quad \text { and } \quad M^{*}=\frac{M}{\mu \pi R^{3}}
$$

are the dimensionless shear force and bending moment in the beam, respectively.

Equation (5.4) can be solved numerically using the method of Liu (1990). We first define the set of $N$ shape functions

$$
w_{i}^{k}(Z)=\left\langle Z-\left(Z_{i}-\frac{1}{2} \delta\right)\right\rangle^{k}-\left\langle Z-\left(Z_{i}+\frac{1}{2} \delta\right)\right\rangle^{k},
$$

where $\delta$ is the dimensionless element length, $Z_{i}=\left(i-\frac{1}{2}\right) \delta, i=1, \ldots, N$,

$$
\langle Z-A\rangle^{k}= \begin{cases}(Z-A)^{k}, & Z>A, \\ 0, & Z<A,\end{cases}
$$

and it can be verified by integration that

$$
\int\langle Z-A\rangle^{k} \mathrm{~d} Z=\frac{\langle Z-A\rangle^{k+1}}{k+1} .
$$

The displacement $U(Z)$ can then be represented in the discrete form

$$
U(Z)=\sum_{i=1}^{N} U_{i} w_{i}^{0}(Z)
$$

Proc. R. Soc. Lond. A (1997) 
and substitution into (5.4), using (5.6), (5.7) yields the system of algebraic equations

$$
\begin{aligned}
\sum_{i=1}^{N}\left(w_{i}^{0}\left(Z_{j}\right)+\frac{C_{1}}{2 h_{i}} w_{i}^{2}\left(Z_{j}\right)-\frac{C_{2}}{24 h_{i}} w_{i}^{4}\left(Z_{j}\right)\right) U_{i} \\
=U(0)+Z_{j} U^{\prime}(0)-\frac{1}{2}\left(C_{2} R Z_{j}^{2}\right) M^{*}(0)-\frac{1}{6}\left(C_{2} R Z_{j}^{3}\right) V^{*}(0) \\
\quad+\sum_{i=1}^{N}\left(\frac{1}{2}\left(C_{1} w_{i}^{2}\left(Z_{j}\right)\right)-\frac{1}{24}\left(C_{2} w_{i}^{4}\left(Z_{j}\right)\right)\right) \frac{U_{A i}}{h_{i}}, \quad j=1, \ldots, N .
\end{aligned}
$$

If the compliance functions $h_{i}$ and the boundary values $U(0), U^{\prime}(0), M^{*}(0), V^{*}(0)$ are assumed known, (5.10) defines $N$ linear equations for the $N$ unknowns $U_{i}$. Equations (4.20), (4.4) can then be used to obtain $F\left(Z_{i}\right), \psi\left(Z_{i}\right)$, respectively, after which (4.21) provides an improved estimate for $h_{i}$. In certain cases, negative values will be obtained for some of the $F\left(Z_{i}\right)$ in this process, indicating the occurrence of local separation (loss of contact). This situation can easily be accommodated in the proposed algorithm by setting the local stiffness $\left(1 / h_{i}\right)$ to zero at separation points.

If the boundary conditions are defined in terms of the end loads $M^{*}(0), V^{*}(0)$, $M^{*}(L), V^{*}(L)$, where $L$ is the dimensionless length of the beam, the rigid body terms in the beam displacement will adopt such values as to ensure that the contact forces $F\left(Z_{i}\right)$ and the end loads are in equilibrium. The equilibrium conditions have the form

$$
\begin{aligned}
\sum_{i=1}^{N} \frac{\delta U_{i}}{R h_{i}} & =-V^{*}(0)+V^{*}(L)+\sum_{i=1}^{N} \frac{\delta U_{A i}}{R h_{i}}, \\
\sum_{i=1}^{N} \frac{\delta U_{i}}{R h_{i}} Z_{i} & =-M^{*}(0)+M^{*}(L)+L V^{*}(L)+\sum_{i=1}^{N} \frac{\delta U_{A i}}{R h_{i}} Z_{i},
\end{aligned}
$$

and these can be used as two additional equations to determine the unknowns $U(0)$, $U^{\prime}(0)$.

\section{(i) Misaligned cylinders}

\section{(b) Examples}

To illustrate the method, we first consider the case of two slightly misaligned identical cylindrical beams of length $L R$ pressed against each other by loads $\frac{1}{2} P$ at their ends, as shown in figure $3 a$. If the misalignment angle $\phi$ is small, the contact kinematics is equivalent to the indentation of a single beam by a rigid cylinder of very large radius $R_{0}$ given by

as shown in figure $3 b$.

$$
\frac{1}{R_{0}}=\frac{\phi^{2}}{4 R}
$$

In the contact region we therefore have

$$
U_{A}=\frac{z^{2}}{2 R_{0}}=\frac{1}{8} \phi^{2} Z^{2} R .
$$

Before presenting the results of the present method, it is helpful to discuss two limiting solutions of the problem given by: (i) the Hertzian contact theory; and (ii) classical beam theory.

Proc. R. Soc. Lond. A (1997) 

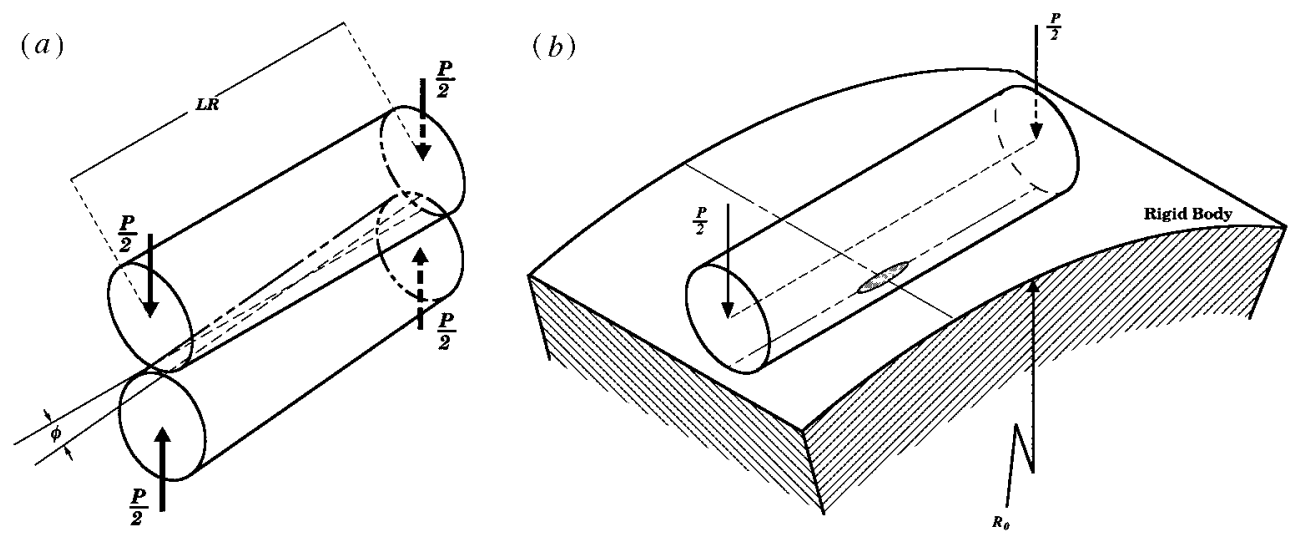

Figure 3. (a) Contact of two identical but misaligned circular cylinders; (b) equivalent contact problem between one cylinder and a rigid body.

The Hertzian solution is given in detail by Barber (1980) and involves a long narrow elliptical contact area. This constitutes a good description of the contact problem as long as the major axis of the ellipse is small compared with the radius $R$ of the cylindrical beam; i.e. at small values of the contact load $P$. It is therefore mathematically exact in the limit $P \rightarrow 0$. However, progressively larger errors are to be anticipated with increasing loads. The principal reason for these errors is that the bending moments in the cylinders cause changes in the local geometry of the contacting surfaces that are significant in comparison with the initial curvatures.

By contrast, the classical beam theory captures the effects of bending, but fails totally to describe the stress fields in the vicinity of the contact area, since it generally predicts that contact is restricted to one or more isolated points. However, we shall see that the location of these points provides a good guide to the nature of the present solution. For the particular problem of figure $3 b$, the beam solution involves a single concentrated force at the contact point for moderate loads and two symmetrically disposed concentrated forces for larger loads. The critical load at which the contact point bifurcates corresponds to that at which the curvature due to the maximum bending moment at the contact point equals that of the indenter and can be shown to be

$$
P_{\mathrm{cr}}=\frac{2 \pi \mu(1+\nu) R^{3}}{R_{0} L}=\frac{\pi \mu(1+\nu) R^{2} \phi^{2}}{2 L}
$$

with the present notation. The distance between the two contact points increases with load in the range $P>P_{\text {cr }}$.

The algorithm of $\S 5 a$ was used to obtain numerical results for a dimensionless length $L=50$ with a misalignment angle $\phi=0.2 \mathrm{rad}\left(=11.5^{\circ}\right)$. Figure 4 shows the evolution of the contact area with increasing load. Notice that the contact areas are very slender and hence scales differing by a factor of 20 have been used in representing the width and the length of the contact area for clarity. In each case, the contact area predicted by the present theory is shown shaded, the corresponding Hertzian contact area is unshaded and the location of the contact point(s) predicted by the elementary beam theory is indicated by arrows. As we would expect, the present theory agrees quite closely with the Hertzian theory for loads well below the critical load, but significant deviation from Hertzian behaviour is observed as the load approaches and passes the critical load. The contact area first becomes disproportionately elongated

Proc. R. Soc. Lond. A (1997) 


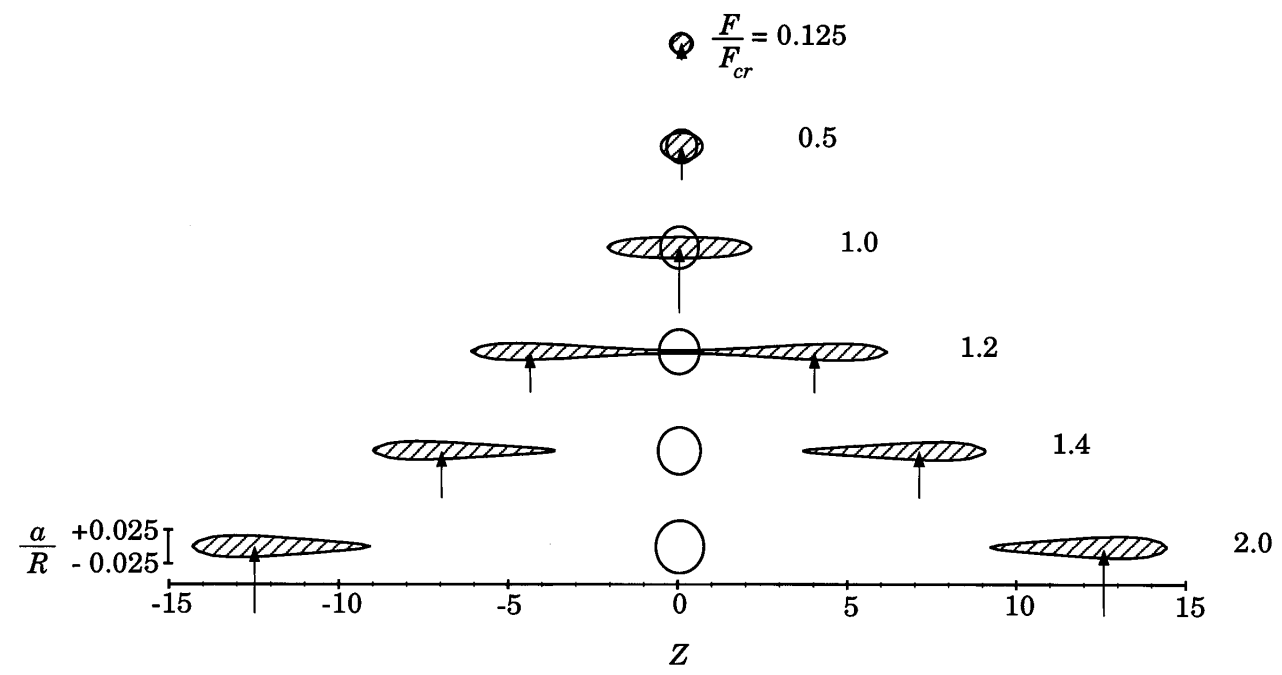

Figure 4. Evolution of the contact area for beams of dimensionless length $L=50$ and Poisson's ratio $\nu=0.3$, misaligned by $\phi=0.2 \mathrm{rad}\left(=11.5^{\circ}\right)$. The contact area predicted by the present theory is shown shaded, the corresponding Hertzian contact area is unshaded and the location of the contact point(s) predicted by the elementary beam theory is indicated by arrows. Notice that the vertical scale is 20 times larger than the horizontal.

in the axial direction, it then assumes a dog-bone shape and finally bifurcates into two symmetrically disposed slender contact areas for $P>1.22 P_{\mathrm{cr}}$. In all cases, the general location of the contact areas is well predicted by the beam theory, but the latter is of course unable to predict the finite length of the contact area and the distribution of contact pressure.

The maximum contact pressure at any given axial location is given by equation (4.1) as

$$
p_{\max }(Z)=\frac{2 F(Z)}{\pi a(Z)}=\frac{\mu a(Z)}{R(1-\nu)},
$$

where we have used (4.4) to eliminate $F(Z)$. Thus, the maximum contact pressure is proportional to the local contact width and figure 4 can therefore also be read as a representation of the maximum contact pressure as a function of $Z$.

Figure 5 shows similar results for a shorter beam (dimensionless length $L=10$ ) with $\phi=0.09 \mathrm{rad}\left(=5.1^{\circ}\right)$. At low loads, the behaviour is similar to that observed in figure 4 , but elongation of the contact area is slower, because the changes in the distribution $F(Z)$ have a proportionally larger effect in reducing the bending moment in the contact region for the shorter beam. Contact extends to the ends of the beam at $P=2.6 P_{\text {cr }}$ and contact is lost at the centre when $P=4.6 P_{\mathrm{cr}}$. Further increase in load then causes a rather slow evolution towards more concentration of load near the ends of the beam.

When contact extends to the ends of the beam, the solution will be correct if and only if the tractions on the ends have the appropriate form. In particular, the end loads must be applied as shear tractions distributed according to equation (2.1) and self-equilibrated normal tractions $\sigma_{z z}$ must be applied so as to preserve the plane strain conditions implied by the two-dimensional solution of $\S 4$. If plane stress conditions $\left(\sigma_{z z}=0\right)$ apply at the ends, some local modification is to be expected in the contact pressure distribution near the ends (Johnson 1985, figure 5.11a).

Proc. R. Soc. Lond. A (1997) 


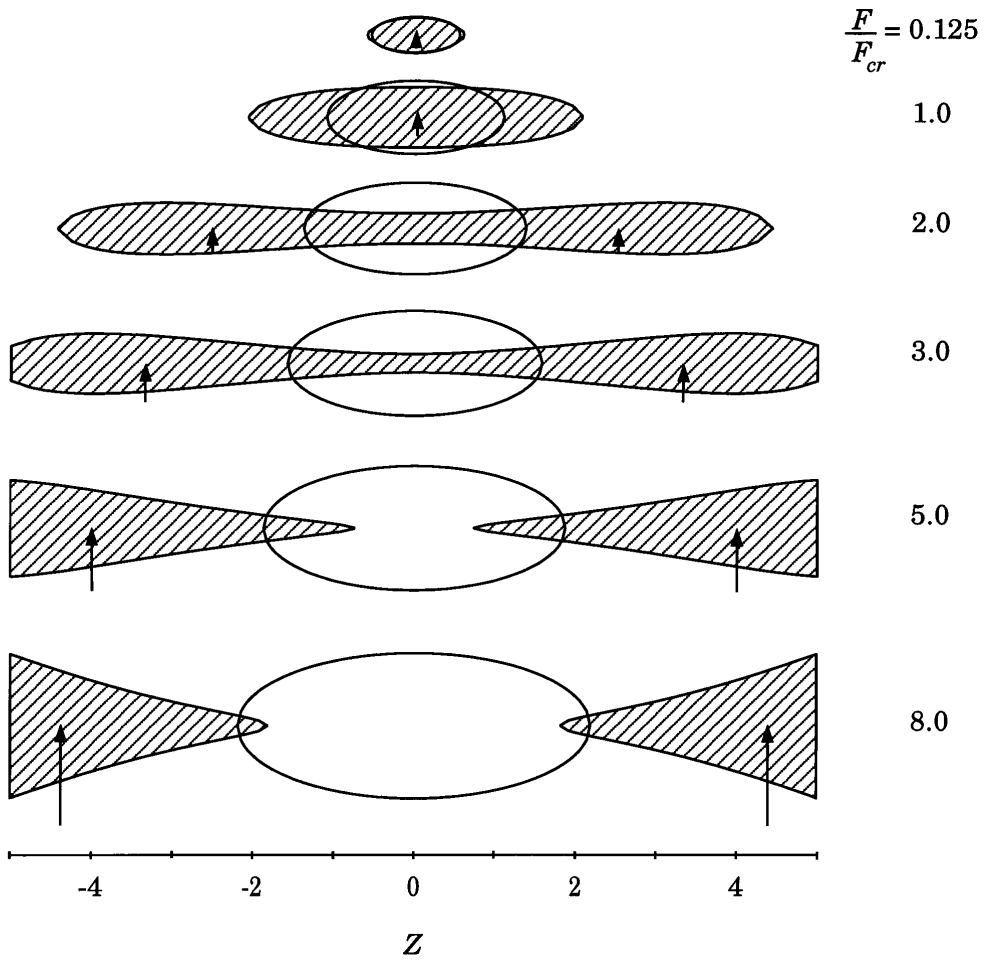

Figure 5. Evolution of the contact area for beams of dimensionless length $L=10$ and Poisson's ratio $\nu=0.3$, misaligned by $\phi=0.09 \mathrm{rad}\left(=5.1^{\circ}\right)$. The contact area predicted by the present theory is shown shaded, the corresponding Hertzian contact area is unshaded and the location of the contact point(s) predicted by the elementary beam theory is indicated by arrows. Notice that the vertical scale is 20 times larger than the horizontal.

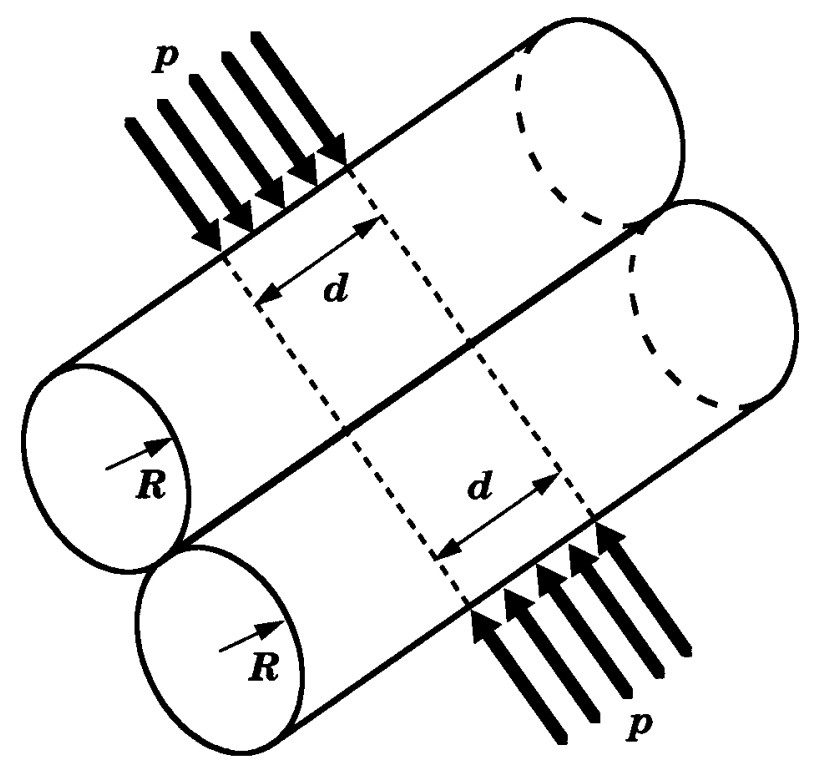

Figure 6. Two identical aligned beams pressed together by a force uniformly distributed over a central region.

Proc. R. Soc. Lond. A (1997) 


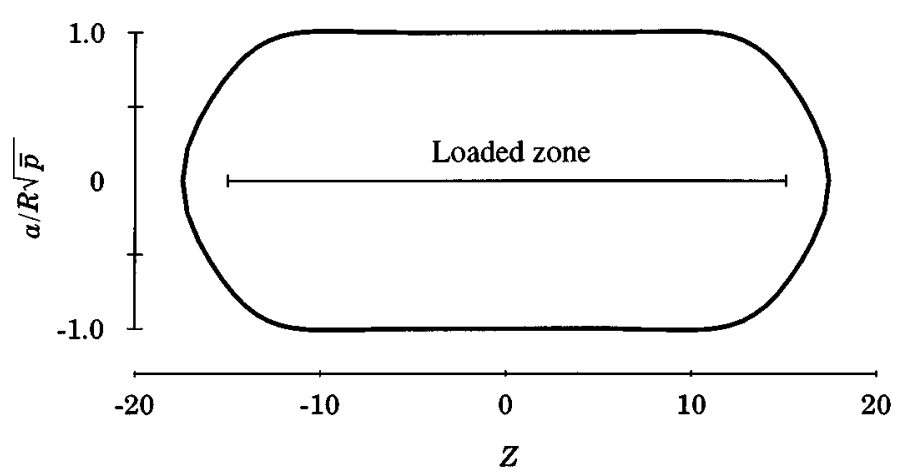

Figure 7. Contact area for the two beams of figure 6 for $\bar{p}=10^{-3}$. Notice that for this load, the vertical dimension of the contact area is magnified about 250 times relative to the horizontal.

\section{(ii) Parallel cylinders with central loading}

As a second example, we consider the case of a pair of identical parallel cylinders of radius $R$, pressed together by a force $p$ per unit length distributed over a central region of length $d$, as shown in figure 6 . Because of symmetry, this is identical to the problem of a single cylinder pressed against a rigid plane.

Figure 7 shows the extent of the contact area for a dimensionless load

$$
\bar{p}=\frac{2 p(1-\nu)}{\pi \mu R},
$$

of $10^{-3}$ and a loaded length $d=30 R$. The cylinders separate at the ends, with contact extending a distance of about $2.4 R$ beyond the end of the loaded region. Increasing the load has virtually no effect on this contact length, but leads to an increase in contact width which is approximately proportional to $\sqrt{\bar{p}}$, as we might expect in view of equation (4.4). Thus figure 7, which presents the contact width $a$ in the combination $a /(R \sqrt{\bar{p}})$, shows almost imperceptible change with load in the range $10^{-5}<\bar{p}<10^{-1}$.

\section{(c) Comparative length scales}

For a beam on a linear elastic foundation of modulus $k$, the axial variation of displacement and support load is characterized by an exponential decay rate of the form $\exp (z / b)$ where

$$
b=\sqrt[4]{\frac{4 E I}{k}} .
$$

If the length of the beam is much greater than $b$, the problem will be dominated by beam bending effects except near the ends or load discontinuities, whereas if the length is much less than $b$, it will be dominated by the elasticity of the foundation and hardly any bending will occur.

In the present problem, the equivalent foundation modulus is not constant, but the relatively weak dependence of the modulus on $\psi$ and the fourth root in equation (5.18) suggests that the length scale of contact-moderated effects will be quite insensitive to local load levels. Figure 8 shows that the decay length lies in the range $1.5 R<b<1.9 R$ for all values of $\nu$ in a three order of magnitude range of the dimensionless local contact load $F / \mu R$.

This result can be used to explain the behaviour observed in figures 4 and 5 at large loads. For example, in figure 4, the separated contact areas have a length of the

Proc. R. Soc. Lond. A (1997) 


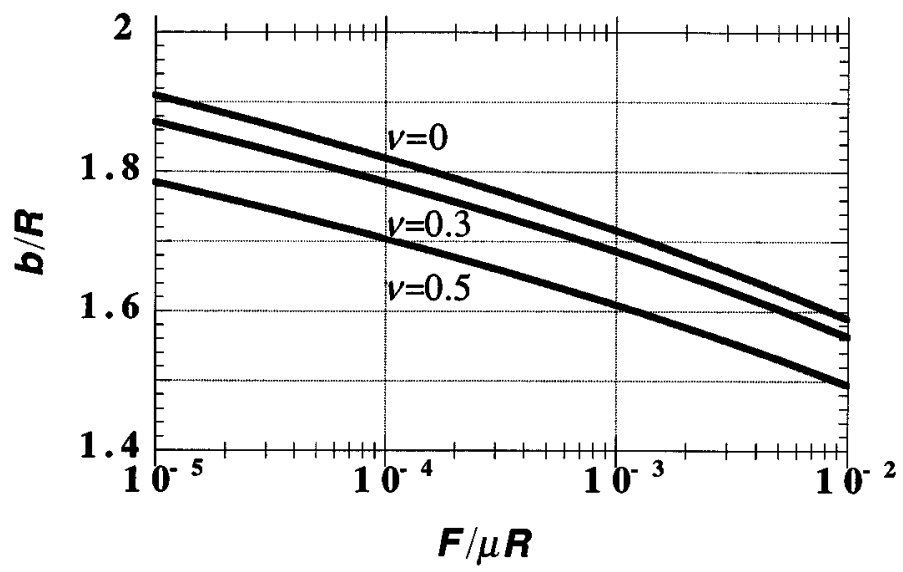

Figure 8. Decay length $b$ as a function of contact load per unit length.

order of $10 R$ and are roughly centred on the predicted location of the concentrated forces in the beam theory. In other words, the concentrated forces are distributed over a range of about $\pm 5 R$ which is about $\pm 3 b$ for this system. By contrast, the half-length of the beam in figure 5 is only about $3 b$ and hence the beam theory does not give a good description of the behaviour even at high loads.

\section{Conclusions}

The above method defines a strategy for accounting both for contact flexibility and beam deflections in problems where a uniform cylindrical beam is loaded through contact either with another beam or with a rigid foundation and where the resulting load varies fairly slowly along the length in comparison with the beam radius. The examples treated demonstrate that both contact and beam bending effects are important in practical cases and that the use of the half-space assumption in the classical Hertzian analysis leads to significant errors when the longitudinal dimension of the contact area is several times larger than the beam radius.

The method is readily extended to problems of beams of non-circular cross-section. All that is required is the solution of an equivalent three-dimensional cantilever problem, to establish the equivalent distribution of $\boldsymbol{f}$ to use in equation (4.5) and the corresponding solution of a plane contact problem for this cross-section to replace the results of $\S 4$.

In more complex examples such as gears or rolls transmitting frictional as well as normal tractions, torsional as well as bending deflections would be needed in the corresponding beam problem, but no new computational difficulties are to be expected.

The authors acknowledge support from the Venezuelan Government through FUNDAYACUCHO (J.C.) and from the National Science Foundation under contract number CMS-9322106 (J.R.B.).

\section{References}

Barber, J. R. 1980 The rolling contact of misaligned elastic cylinders. J. Mech. Engng Sci. 22, $125-128$.

Barber, J. R. 1992 Elasticity. Dordrecht: Kluwer.

Proc. R. Soc. Lond. A (1997) 
Bennon, W. D. 1985 Evaluation of selective coolant application for the control of work roll thermal expansion. ASME J. Engng Industry 107, 146-152.

Dundurs, J. 1975 Properties of elastic bodies in contact. In The mechanics of the contact between deformable bodies (ed. A. D. de Pater \& J. J. Kalker), pp. 54-66. Delft University Press.

Johnson, K. L. 1985 Contact mechanics. Cambridge University Press.

Juvinall, R. C. 1983 Fundamentals of machine component design. Figure 9.18a. New York: Wiley.

Kalker, J. J. 1972 On elastic line contact. ASME J. Appl. Mech. 39, 1125-1132.

Kalker, J. J. 1977 The surface displacement of an elastic half-space loaded in a slender bounded curved surface region with application to the calculation of the contact pressure under a roller. J. Inst. Math. Appl. 19, 127-144.

Liu, Shin-Ya 1990 Elastic-plastic contact analysis of laterally loaded piles. Ph.D. thesis, University of Michigan.

Love, A. E. H. 1927 A treatise on the mathematical theory of elasticity, 4th edn. Cambridge University Press.

McDonald, I. R. \& Spooner, P. D. 1989 The effect of mill design on strip quality,. In Modernization of steel rolling. Proc. Int. Conf. on Modernization of Steel Rolling, pp. 613-620. Oxford: Pergamon.

Persson, A. 1964 On the stress distribution of cylindrical elastic bodies in contact. Dissertation, Chalmers Tekniska Hogskola, Göteborg.

Pilkey, W. D. 1994 Formulas for stress, strain and structural matrices, p. 1328. New York: Wiley.

Renton, J. D. 1991 Generalized beam theory applied to shear stiffness. Int. J. Solids Struct. 27, 1955-1967.

Renton, J. D. 1996 Generalized beam theory and modular structures. Int. J. Solids Struct. 33, $1425-1438$.

Timoshenko, S. P. \& Goodier, J. N. 1970 Theory of elasticity, 3rd edn. New York: McGraw-Hill.

Received 23 December 1996; accepted 30 May 1997

Proc. R. Soc. Lond. A (1997) 